\title{
Imaging Mitochondrial Redox Environment and Oxidative Stress Using a Redox-Sensitive Fluorescent Protein
}

Alexander M. Wolf, Sadamitsu Asoh, Ikuroh Ohsawa and Shigeo Ohta Department of Biochemistry and Cell Biology, Institute of Development and Aging Sciences, Graduate School of Medicine, Nippon Medical School

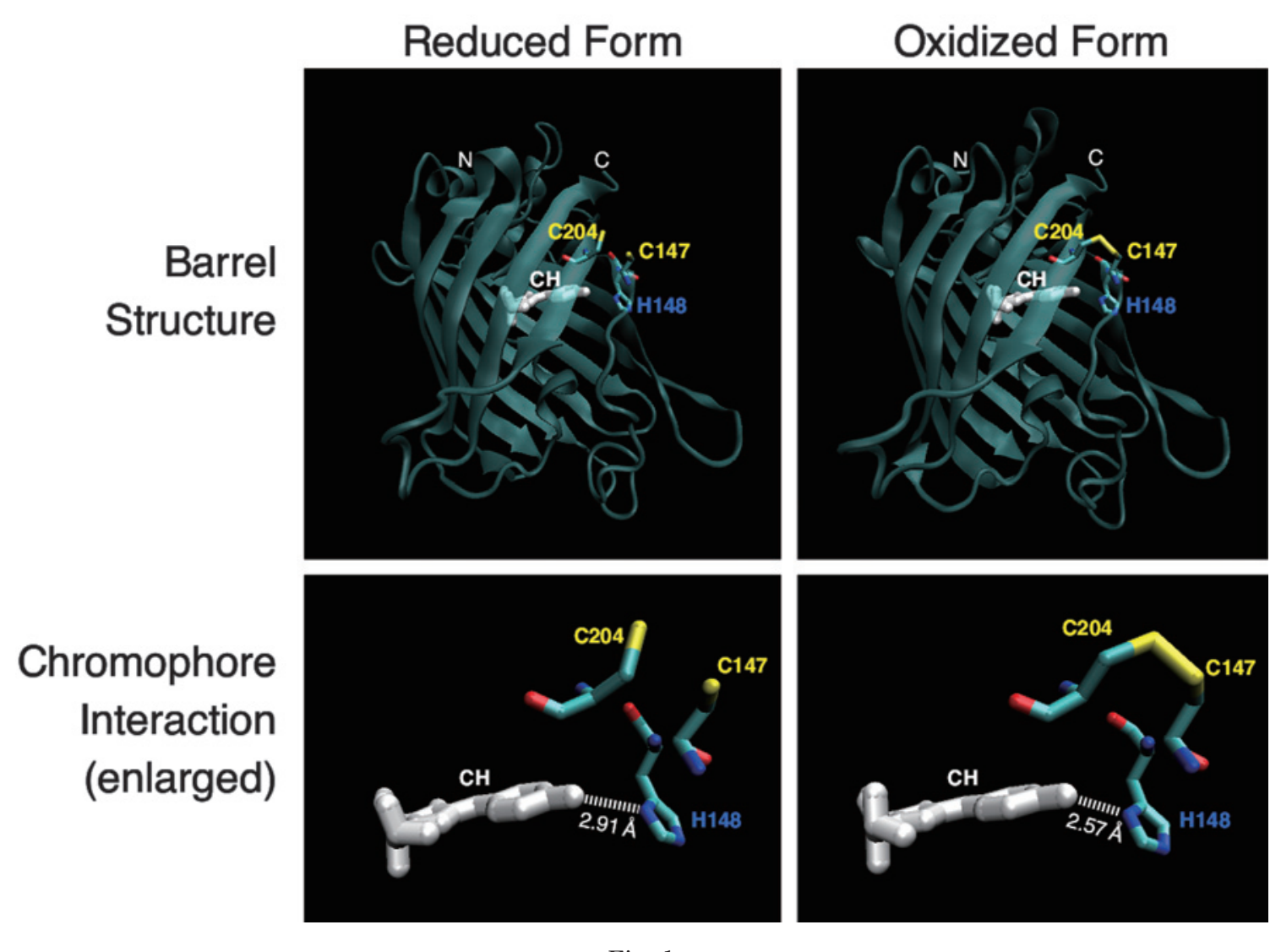

Fig. 1

\section{Abstract}

Redox-sensitive green fluorescent protein (roGFP) is a fluorescent protein in which two cysteines are placed adjacently in the barrel structure. Disulfide formation (oxidation) increases the absorption at short wavelengths $(410 \mathrm{~nm})$ at the expense of absorption at longer wavelengths $(490 \mathrm{~nm})$. The fluorescence ratio indicates reduction/oxidation, i.e., the redox potential at specific cellular locations.

Correspondence to Sadamitsu Asoh, Department of Biochemistry and Cell Biology, Institute of Development and Aging Sciences, Graduate School of Medicine, Nippon Medical School, 1-396 Kosugi-cho, Nakahara-ku, Kawasaki, Kanagawa 211-8533, Japan 


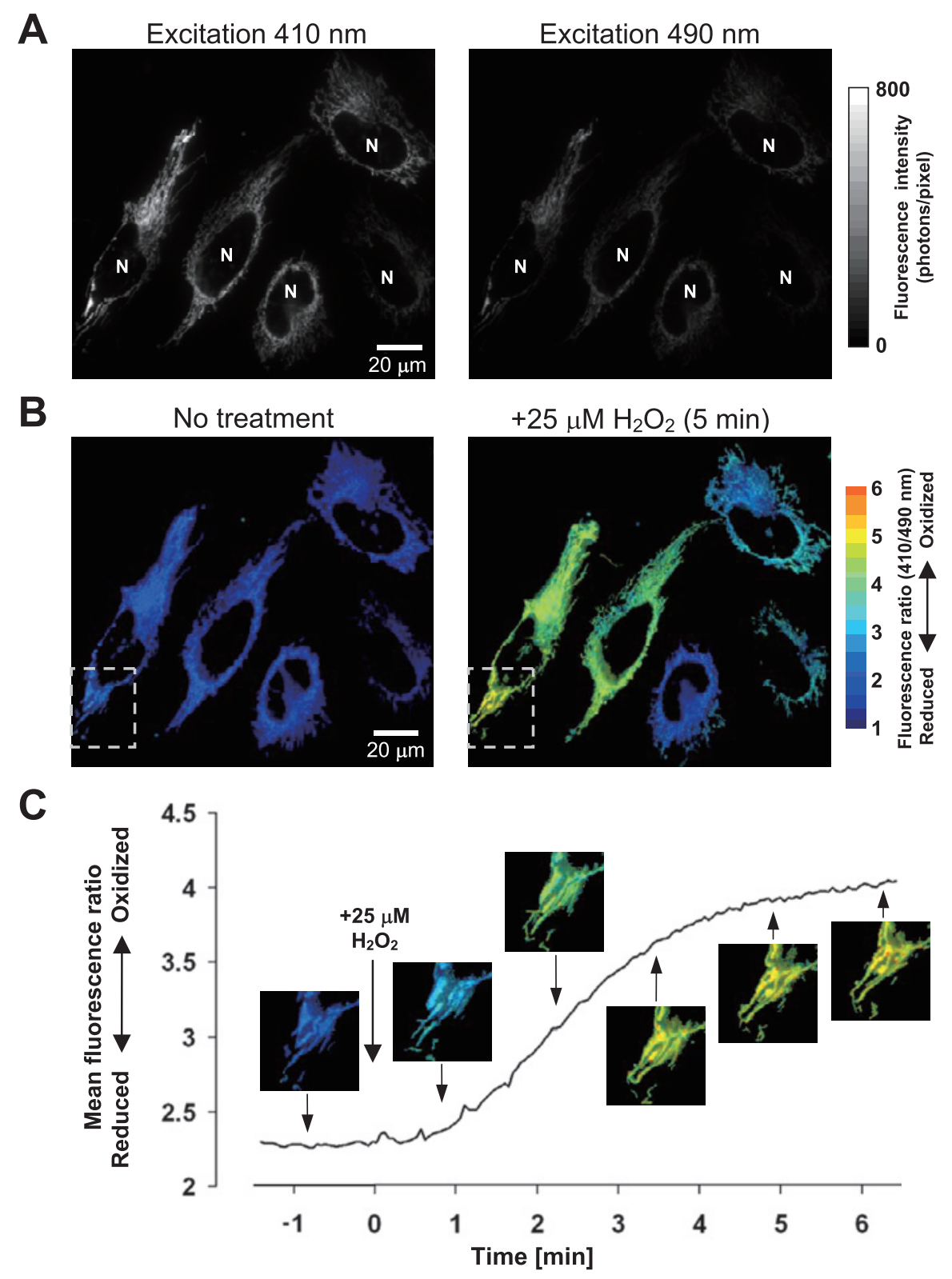

Fig. 2

Fig. 1 Rendered visualization of the reduced and oxidized roGFP barrel structure (green, semitransparent) protecting the chromophore $(\mathrm{CH}$, white), the location of the two cysteines (C147 and C204), and the histidine (H148) involved in the chromophore interaction. The enlargement shows how the formation of a disulfide bridge (yellow) brings histidine 148 closer to the chromophore, changing the absorption spectrum of the protein.

Fig. 2 (A) Fluorescence images of HeLa cells expressing roGFP targeted to the mitochondrial matrix (N: nucleus). roGFP excitation is higher at $410 \mathrm{~nm}$. (B) Visualization of redox state by dividing fluorescence intensities and assigning color according to a scale. Hydrogen peroxide induced oxidation of the mitochondrial matrix. (C) Time course of the mean fluorescence ratio of the cells in $\mathrm{B}$, showing gradual matrix oxidation after addition of $\mathrm{H}_{2} \mathrm{O}_{2}$. Inset magnifications (boxed area in $\mathrm{B}$ ) show the dynamic shift of the mitochondrial redox potential.

E-mail: sada@nms.ac.jpＪournal Website (http://www.nms.ac.jp/jnms/) 